\title{
C/EBP homologous protein modulates liraglutide- mediated attenuation of non-alcoholic steatohepatitis
}

\author{
Khalidur Rahman ${ }^{1,2,4}$, Yunshan Liü ${ }^{2,4}$, Pradeep Kumar ${ }^{1}$, Tekla Smith ${ }^{1,2}$, Natalie E Thorn ${ }^{1}$, Alton B Farris ${ }^{3}$ and \\ Frank A Anania ${ }^{1,2}$
}

The CCAAT/enhancer-binding protein (C/EBP) homologous protein (CHOP), a major transcriptional regulator of endoplasmic reticulum (ER) stress-mediated apoptosis, is implicated in lipotoxicity-induced ER stress and hepatocyte apoptosis in non-alcoholic fatty liver disease (NAFLD). We have previously demonstrated that the glucagon-like peptide-1 (GLP-1) agonist, liraglutide, protects steatotic hepatocytes from lipotoxicity-induced apoptosis by improved handling of free fatty acid (FFA)-induced ER stress. In the present study, we investigated whether CHOP is critical for GLP-1-mediated restoration of ER homeostasis and mitigation of hepatocyte apoptosis in a murine model of NASH (non-alcoholic steatohepatitis). Our data show that despite similar caloric intake, $\mathrm{CHOP} \mathrm{KO}\left(\mathrm{CHOP}^{-/-}\right)$mice fed a diet high in fat, fructose, and cholesterol (HFCD) for 16 weeks developed more severe histological features of NASH compared with wild-type (WT) controls. Severity of NASH in HFCD-fed $\mathrm{CHOP}^{-1-}$ mice correlated with significant decrease in peroxisomal $\beta$-oxidation, and increased de novo lipogenesis and ER stress-mediated hepatocyte apoptosis. Four weeks of liraglutide treatment markedly attenuated steatohepatitis in HFCD-fed WT mice by improving insulin sensitivity, and suppressing de novo lipogenesis and ER stress-mediated hepatocyte apoptosis. However, in the absence of CHOP, liraglutide did not improve insulin sensitivity, nor suppress peroxisomal $\beta$-oxidation or ER stress-mediated hepatocyte apoptosis. Taken together, these data indicate that CHOP protects hepatocytes from HFCD-induced ER stress, and has a significant role in the mechanism of liraglutidemediated protection against NASH pathogenesis.

Laboratory Investigation (2016) 96, 895-908; doi:10.1038/labinvest.2016.61; published online 30 May 2016

Non-alcoholic fatty liver disease (NAFLD) represents a broad spectrum of disorders including benign non-alcoholic fatty liver or bland steatosis (NAFL), to the more severe non-alcoholic steatohepatitis (NASH) and steatofibrosis. ${ }^{1,2}$ According to recent reports, the prevalence of NAFL ranges from 20 to $30 \%$ in the general population and as high as $75-100 \%$ in obese individuals. ${ }^{3,4}$ Although most NAFL patients remain asymptomatic, one fifth of NAFL patients progress to chronic hepatitis (NASH), which can further progress to cirrhosis, portal hypertension, and promote development of hepatocellular carcinoma. ${ }^{4,5}$ Despite its high prevalence, and established morbidity and mortality associated with NAFLD, mechanisms leading to NAFLD progression remain poorly understood.

Systemic insulin resistance, lipotoxicity, and oxidative stress are central players in the pathogenesis of NASH. ${ }^{6-8}$ Recent studies indicate that the unfolded protein response
(UPR), an adaptive response that regulates ER function during ER stress through transcriptional and translational modulation of factors involved in ER homeostasis, also has a critical role in free fatty acid (FFA) and free-cholesterolmediated lipotoxicity. ${ }^{8-14}$ Under conditions of nutrient overload, excess FFA and cholesterol induce activation of the UPR. ${ }^{9,14}$ Failure of the UPR to maintain ER homeostasis in the setting of metabolic stress, resulting from nutrient overload, leads to ER stress-mediated hepatocyte apoptosis. ${ }^{1,8,10,11,15-18}$ Therefore, strategies to prevent ER stress-related hepatocyte death are highly desirable and would likely halt progression of NAFL to NASH.

Accumulating evidence suggests that CCAAT/enhancerbinding protein (C/EBP) homologous protein (CHOP) is a major transcriptional regulator of ER stress-mediated apoptosis. ${ }^{19,20}$ CHOP is implicated in a wide array of common diseases including neurodegenerative, cardiovascular,

\footnotetext{
${ }^{1}$ Division of Digestive Diseases, Department of Medicine, Emory University, Atlanta, GA, USA; ${ }^{2}$ Atlanta VA Medical Center, Decatur, GA, USA and ${ }^{3}$ Department of Pathology, Emory University Hospital, Atlanta, GA, USA

Correspondence: Dr K Rahman, PhD, Digestive Diseases, Department of Medicine, Emory University School of Medicine, 615 Michael Street, Suite 201, Atlanta, GA 30322, USA. E-mail: reben.rahman@emory.edu

${ }^{4}$ These authors contributed equally to this work.

Received 9 November 2015; revised 15 April 2016; accepted 26 April 2016
} 
and metabolic disorders, including NAFLD. ${ }^{21-27}$ Apart from modulating ER stress-mediated apoptosis, CHOP also has a role in transcriptional regulation of cellular lipid metabolism ${ }^{20,28}$ suggesting that increased hepatic expression of CHOP in NAFLD patients may protect hepatocytes from FFA and cholesterol induced lipotoxicity. However, a mechanism whereby $\mathrm{CHOP}$ mediates protection against FFA-induced ER stress is not entirely clear.

We and others have demonstrated a beneficial role for glucagon-like peptide-1 (GLP-1) for NAFLD treatment. ${ }^{29-34}$ GLP-1 is an incretin hormone secreted by the L-cells of the distal small intestine and proximal colon. ${ }^{35}$ Prior data indicate that GLP-1 analogs can suppress FAA-induced steatosis in isolated hepatocytes, and in animal models of NASH fed a diet high in carbohydrate, saturated fat, and fructose. A recent small-scale double-blind placebo-controlled clinical trial in which the long-acting GLP-1 analog, liraglutide was tested demonstrated liraglutide reduced metabolic dysfunction, increased hepatic insulin sensitivity, and reduced hepatic de novo lipogenesis (DNL) in vivo. ${ }^{36}$ Studies in mice and cell culture models of steatosis demonstrate that GLP-1 agonists, liraglutide or exendin-4, protect steatotic hepatocytes from apoptosis by improved handling of FFA-induced ER stress. ${ }^{31,34}$ Here, by using global knockout mice for the critical ER stress protein, CHOP, we provide in vivo and in vitro evidence that liraglutide mediates its protective effects through CHOP to ultimately prevent hepatocyte apoptosis and NAFLD progression. Our results demonstrate that in the absence of CHOP, liraglutide administration fails to ameliorate steatohepatitis in mice fed a HFCD rendering hepatocytes far more susceptible to death.

\section{MATERIALS AND METHODS}

\section{Mice}

$\mathrm{CHOP}^{-/-}$mice on a C57BL/6 background were purchased from Jackson Laboratories (Bar Harbor, ME, USA). Animals were housed in standard micro-isolator cages and maintained on a $12 \mathrm{~h}: 12 \mathrm{~h}$ light/dark cycle. All animals received humane care and all procedures were approved by the Institutional Animal Care and Use Committee of the Veterans' Administration Hospital in Decatur, GA, USA.

\section{Diet}

Five-week-old, age and weight-matched, adult male mice were fed a high-fat, high-cholesterol diet and high-fructose diet (HFCD) containing $0.2 \%$ cholesterol, $20 \%$ protein, $43 \%$ $\mathrm{CHO}, 23 \%$ fat $(6.6 \%$ trans-fat $)$ and $2.31 \%$ fructose (TD.130885; Harlan Laboratories); 37,38 or the standard diet (ND) containing $16 \%$ protein, $61 \%$ carbohydrate and $7.2 \%$ fat. All cohorts were fed ad libitum for 16 weeks. After 12 weeks of feeding, mice received daily intraperitoneal injections of saline or liraglutide ( $200 \mu \mathrm{g} / \mathrm{kg}$ body weight) for 4 weeks. Mice were weighed weekly and food intake per cage was also measured weekly. At the end of the 16 weeks, mice were fasted for $8 \mathrm{~h}$ before euthanasia.

\section{Histopathology and Immunohistochemistry}

Formalin-fixed liver tissues were paraffin-embedded, sectioned, and stained with H\&E, Sirius Red, and Oil Red O as described previously. ${ }^{37}$ Sirius Red-stained areas were quantified by ImageJ software. ${ }^{39}$ NASH scoring was performed by a liver pathologist using metrics for the NASH-CRN. ${ }^{40}$ Apoptosis was detected using a terminal deoxynucleotidyl transferase dUTP nick end-labeling (TUNEL) assay as per the manufacturer's instructions (R\&D Systems, Minneapolis, MN, USA). For immunohistochemistry, paraffin-embedded liver tissue sections were probed with $\mathrm{F} 4 / 80$ antibody (eBiosciences, San Diego, CA, USA), followed by detection with HRP-conjugated secondary antibody and DAB substrate kit (Cell Signaling, Danvers, MA, USA) following manufacturer's guidelines. Photomicrographs of histologic sections were obtained using a Zeiss Light Microscope (Zeiss, Jena, Germany).

\section{Serological Analysis}

Blood obtained by cardiac puncture was collected for measurements of serum alanine aminotransferase (ALT), and aspartate aminotransferase (AST) concentrations using an AST and ALT Activity Assay Kit (Sigma-Aldrich, St Louis, MO, USA).

\section{Quantitative Real-Time PCR}

Isolation of total RNA from liver, cDNA synthesis and qRT-PCR were performed as previously described. ${ }^{41}$ Expression data were normalized to $18 \mathrm{~S}$ rRNA and data are presented as fold change in gene expression compared to WT- standard chow (ND) fed controls.

\section{Glucose Tolerance Test and Insulin Tolerance Test}

Glucose tolerance tests and insulin tolerance tests were performed at baseline, and after 16 weeks of HFCD feeding as described previously. ${ }^{30}$ Blood glucose concentration was measured using a hand held glucometer (Freestyle Flash, Abbott Laboratories, Abbott Park, IL, USA) as described elsewhere. ${ }^{30}$

\section{Immunoblotting}

Liver tissues were homogenized and sonicated in RIPA lysis buffer (Sigma-Aldrich) containing protease inhibitor cocktail and phosphostop (Roche Diagnostics, Indianapolis, IN, USA), and total protein was extracted by centrifugation. A total of $30 \mu \mathrm{g}$ protein was resolved on $4-12 \%$ precast SDS-PAGE gel (Invitrogen, Grand Island, NY, USA) and transblotted onto a polyvinylidene fluoride membrane (Bio-Rad, Hercules, CA, USA). Membranes were probed with antisera against p-JNK,

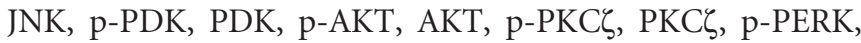
PERK, p-elF2 $\alpha$, elF2 $\alpha$, p-IRE-1, IRE-1, GRP78, CHOP, caspase-3, cleaved caspase-3, ATF-6 $\alpha$, XBP-1, BCL-2, Bax, LC-3B and $\beta$-actin (Cell Signaling Technology, Danvers, MA, USA). Isotype-matched horseradish peroxidase conjugated secondary antibodies, enhanced chemiluminescence substrate 
(Pierce, Rockford, IL, USA) and a FluorChem 8900 digital imaging system (AlphaInnotech, San Leandro, CA, USA) were used to visualize protein bands. Densitometric analyses was performed with VisionWorks Software, version 6.8 (UVP, Upland, CA, USA).

\section{Hepatic Hydroxyproline Quantification}

Hepatic 4-hydroxyproline concentration was quantified using the Hydroxyproline Assay kit (Sigma-Aldrich) following manufacturer's guidelines.

\section{Primary Mouse Hepatocyte Isolation and In Vivo Fatty Acid and Exendin-4 Treatment}

Primary mouse hepatocytes from 8 weeks old WT and $\mathrm{CHOP}^{-/-}$mice were isolated as previously described. ${ }^{42}$ Isolated hepatocytes were cultured in William's medium E Formulation (Sigma-Aldrich) supplemented with 10\% fetal bovine serum (FBS; Atlanta Biologicals, Flowery Branch, GA, USA) and 5\% penicillin-streptomycin (Sigma-Aldrich). The in vitro fatty acid and exendin-4 treatments were performed as described previously. ${ }^{31}$ Hepatocytes were pretreated with $400 \mu \mathrm{M}$ palmitic acid (PA) in ethanol (EtOH) for $24 \mathrm{~h}$, after which hepatocytes were treated with $10 \mathrm{nM}$ exendin- 4 and PA for an additional $24 \mathrm{~h}$. Hepatocytes treated with $\mathrm{EtOH}$ (control), or EtOH plus exendin-4 (control+Ex4), or PA alone were used as controls. At the end of the experiment, cells were subjected to RNA isolation or Oil Red O staining as described above. Gene expression data were normalized to GAPDH and data are presented as fold change in gene expression compared with WT+EtOH controls.

\section{Statistical Analysis}

Values were reported as means \pm s.e.m. and each treatment group included 5-7 mice. All experiments were repeated at least two times on two separate occasions. Data were analyzed by analysis of variance (ANOVA) with Bonferroni's post hoc test for determination of statistical significance $(P<0.05)$.

\section{RESULTS \\ Liraglutide Treatment Attenuates Steatohepatitis in HFCD-Fed WT Mice}

We have previously demonstrated that liraglutide treatment significantly reduced steatosis in WT mice fed a high-fat and high-sugar diet (HFD). ${ }^{30-34}$ We recently reported that mice fed $0.2 \%$ cholesterol in addition to HFD (HFCD) for 16 weeks resulted in mild steatohepatitis characterized by steatosis, inflammation, and mild fibrosis. ${ }^{37}$ Here, we first determined whether liraglutide treatment would also be effective in suppressing steatohepatitis in HFCD-fed mice. As shown in Figure $1 \mathrm{a}-\mathrm{m}$, WT mice fed the HFCD for 16 weeks developed histological features of steatohepatitis indicated by ballooning degeneration of hepatocytes, inflammatory cell infiltration and sinusoidal fibrosis. In agreement with previous reports, ${ }^{30-34} 4$ weeks of liraglutide treatment significantly reduced hepatic steatosis, body weight, and both liver and visceral fat weight expressed as percent of body weight (Figure 1a-f). Liraglutide treatment also significantly reduced the NASH-CRN histology score, ${ }^{43}$ hepatic hydroxyproline levels, and serum AST and ALT levels (Figure 1g-j).

\section{CHOP Modulates Liraglutide-Mediated Suppression of Steatohepatitis}

As anticipated, $\mathrm{CHOP}$ expression was markedly increased in HFCD-fed WT mice, and the daily administration of liraglutide suppressed $\mathrm{CHOP}$ expression (Figure 6a and h). Therefore, we investigated whether $\mathrm{CHOP}$ was pivotal in liraglutide-mediated suppression of steatohepatitis. Consistent with previous observations, ${ }^{44} \mathrm{HFCD}$-fed $\mathrm{CHOP}^{-/-}$mice developed more severe histological features of NASH compared with HFCD-fed WT mice (Figure $1 \mathrm{a}-\mathrm{c}$ and g, and Figure $2 \mathrm{a}-\mathrm{c}$ and g). HFCD-fed $\mathrm{CHOP}^{-/-}$mice gained greater body weight, and had greater liver weight and visceral fat weight compared with HFCD-fed WT mice (Figure 1d-f and Figure 2d-f). HFCD-fed $\mathrm{CHOP}^{-/-}$mice had higher hepatic fibrosis compared with HFCD-fed WT mice (Figure $1 \mathrm{k}-\mathrm{m}$ and Figure $2 \mathrm{k}-\mathrm{m}$ ). In addition, hepatic inflammation in HFCD-fed $\mathrm{CHOP}^{-/-}$mice was higher than HFCD-fed WT mice as evidenced by increased infiltration of macrophages, increase in tumor necrosis factor (TNF)- $\alpha$ and monocyte chemoattractant protein transcript levels, and higher serum AST and ATL levels (Figure $3 \mathrm{a}-\mathrm{c}$ and Figure 2a, g and $\mathrm{m}$ ). Liraglutide treatment attenuated steatosis and hepatic inflammation in HFCD-fed $\mathrm{CHOP}^{-/-}$mice, but this effect was far less robust compared with the liraglutide-treated HFCD-fed WT mouse cohort (Figure 1a-m; Figure 2a-m and Figure $3 \mathrm{a}-\mathrm{c}$ ). Taken together, these data suggest that $\mathrm{CHOP}$ serves to protect mouse liver from HFCD-mediated hepatic inflammation. Also, that $\mathrm{CHOP}$ has an essential role in liraglutide-mediated protection from $\mathrm{NASH}$-associated liver injury.

\section{HFCD-Fed $\mathrm{CHOP}^{-/-}$Mice are Resistant to Liraglutide- Mediated Improvement in Insulin Sensitivity and Glucose Homeostasis}

We and others have previously demonstrated that liraglutide treatment attenuates steatosis by improving hepatic insulin sensitivity in both in vitro and in vivo models of hepatic steatosis. ${ }^{30-34}$ As CHOP has a role in suppressing metabolic genes during ER stress, ${ }^{20,28}$ we tested whether liraglutide improves insulin sensitivity in the absence of CHOP. As seen in Figure $4 \mathrm{a}-\mathrm{d}$, liraglutide treatment significantly improved hyperglycemia and insulin sensitivity in HFCD-fed WT mice. However, HFCD-fed $\mathrm{CHOP}^{-/-}$mice remained insulin resistant and hyperglycemic despite 4 weeks of liraglutide treatment (Figure 4a-d). To determine the molecular mechanisms that could account for persistent insulin resistance in the $\mathrm{CHOP}^{-/-}$mice, we performed a detailed analysis of the downstream mediators of the insulin signaling pathway. As shown in Figure $4 \mathrm{e}-\mathrm{j}$, we demonstrated that activation of PDK, AKT, and PKCל were significantly 
a

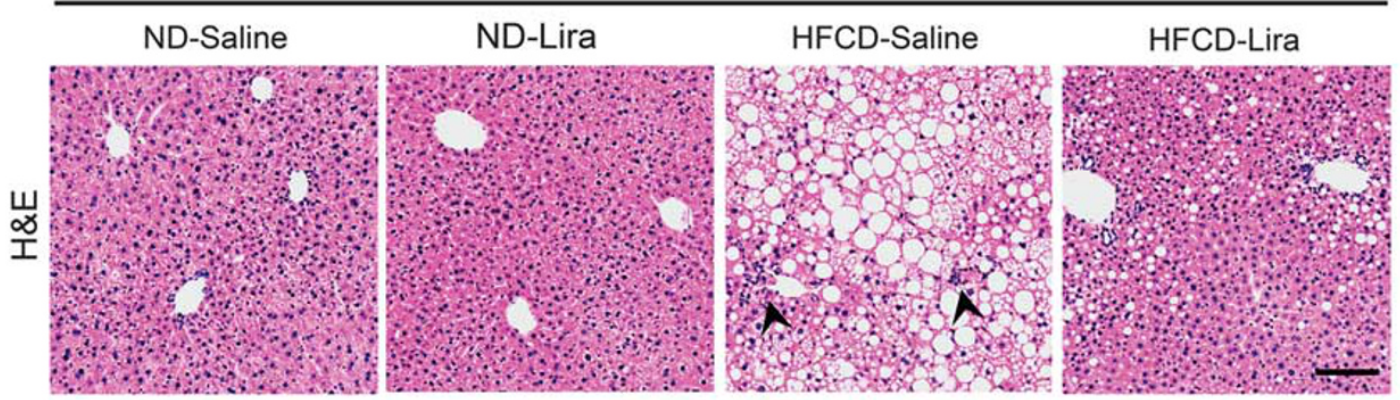

b

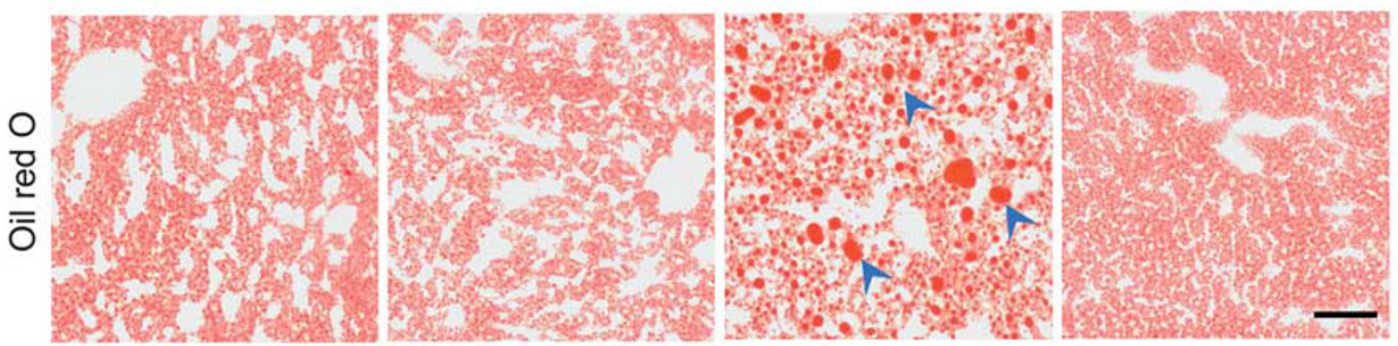

C
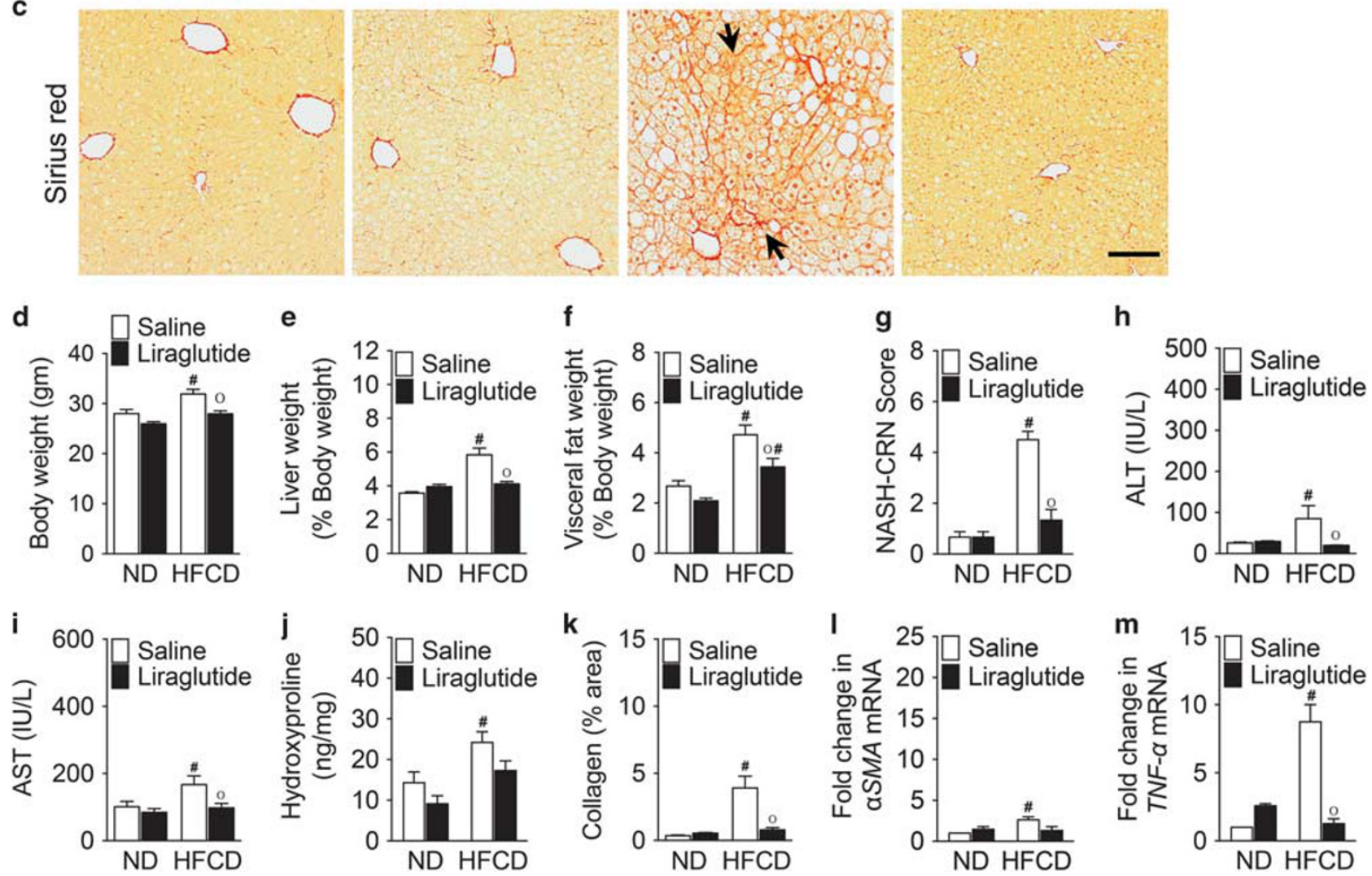

Figure 1 Liraglutide treatment attenuates steatohepatitis in HFCD-fed WT mice. Photomicrographs of (a) Hematoxylin and Eosin, (b) Oil Red O, and (c) Sirius Red-stained liver sections of WT mice fed a ND or HFCD for 16 weeks. After 12 weeks of feeding, mice received daily injections of saline or liraglutide (Lira) for 4 weeks. Black arrowheads, inflammatory immune cells; blue arrowheads, fat droplets; black arrows, collagen deposition. (d) Body, (e) liver, and (f) visceral fat weight as well as (g) NASH-CRN score, serum (h) ALT and (i) AST levels, and (j) hepatic hydroxyproline levels measured at the end of the 16-week experiments. Changes in liver and visceral fat weights are reported as percentages of body weight. (k) Quantitative analysis of Sirius Red-stained liver tissue sections. Quantitative RT-PCR analysis of hepatic (I) aSMA and (m) TNF-a transcript levels measured at the end of the 16 -week experiments. Data are presented as mean \pm s.e.m.; $n=5-7$ mice per group. Hashtags indicate significant differences $(P<0.05)$ between ND- or HFCD-fed WT mice. ' $o$ ' indicates significant differences $(P<0.05)$ between saline- or liraglutide-treated WT mice. Scale bar, $20 \mu \mathrm{m}$. 


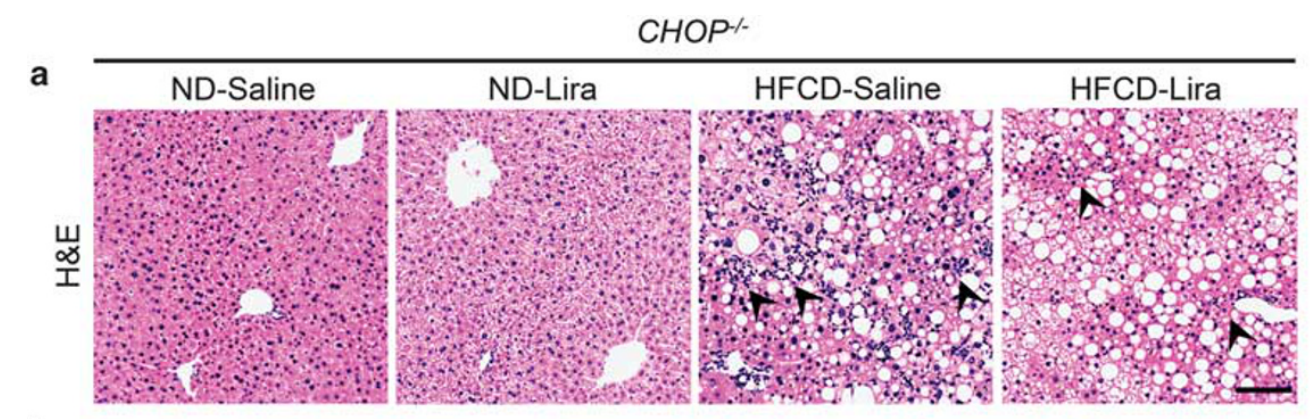

b

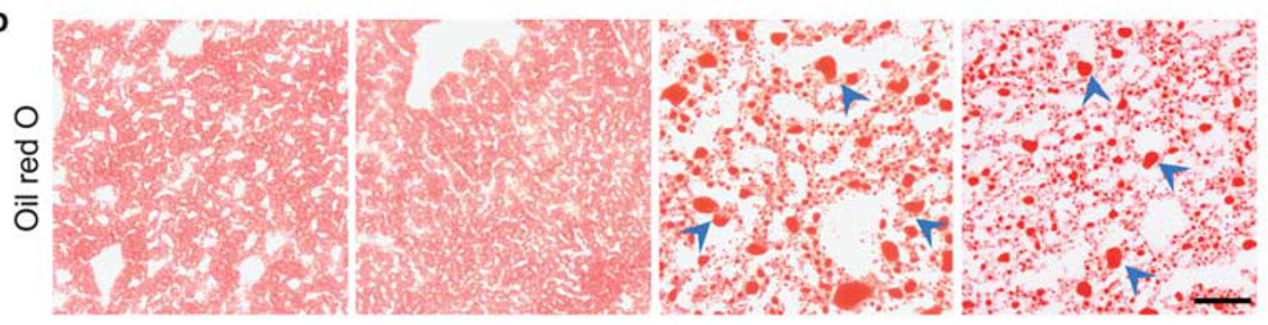

C

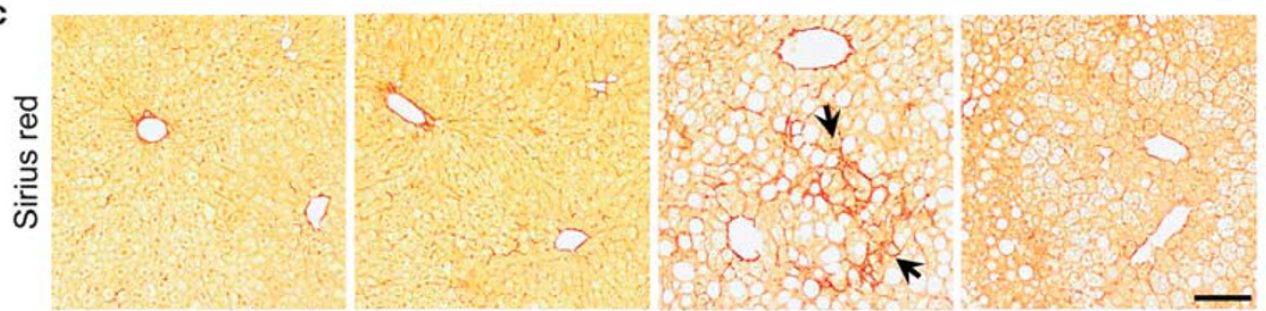

d
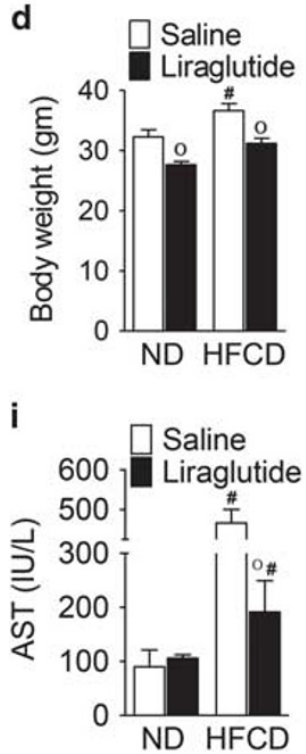

e

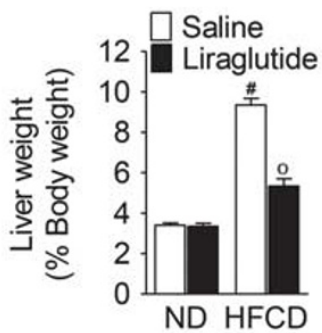

j

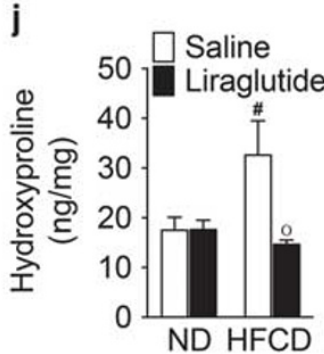

f

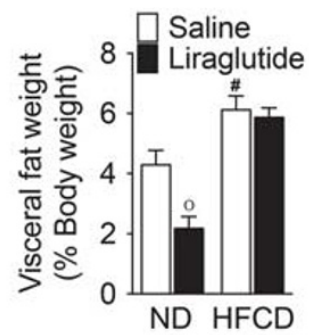

k

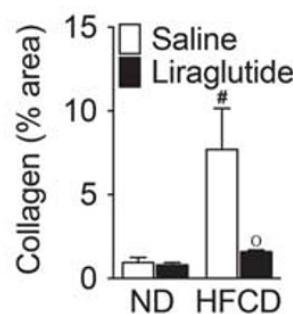

g

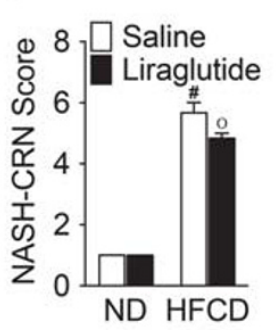

I

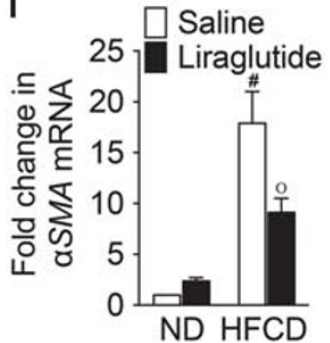

h

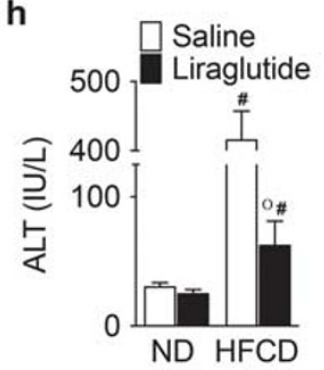

m

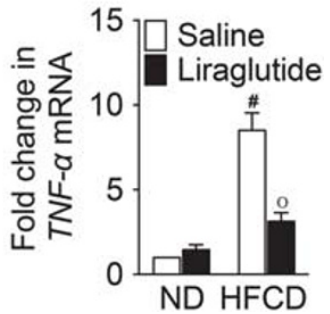

Figure 2 CHOP modulates liraglutide-mediated suppression of steatohepatitis. Photomicrogaphs of (a) Hematoxylin and Eosin, (b) Oil Red O, and (c) Sirius Red-stained liver tissue sections of $\mathrm{CHOP}^{-/-}$mice fed a ND or HFCD for 16 weeks. After 12 weeks of feeding, mice received daily injections of saline or liraglutide (Lira) for 4 weeks. Black arrowheads, inflammatory immune cells; blue arrowheads, fat droplets; black arrows, collagen deposition. (d) Body, (e) liver, and (f) visceral fat weight as well as (g) NASH-CRN score, serum (h) ALT and (i) AST levels, and (j) hepatic hydroxyproline levels measured at the end of the 16-week experiments. Changes in liver and visceral fat weights are reported as percentages of body weight. (k) Quantitative analysis of Sirius Red-stained liver tissue sections. Quantitative RT-PCR analysis of hepatic (I) aSMA and ( $\mathbf{m})$ TNF-a transcript levels measured at the end of the 16-week experiments. Data are presented as mean \pm s.e.m.; $n=5-7$ mice per group. Hashtags indicate significant differences $(P<0.05)$ between ND- or HFCD-fed WT mice. 'o' indicates significant differences $(P<0.05)$ between saline- or liraglutide-treated WT mice. Scale bar, $20 \mu \mathrm{m}$. 
a
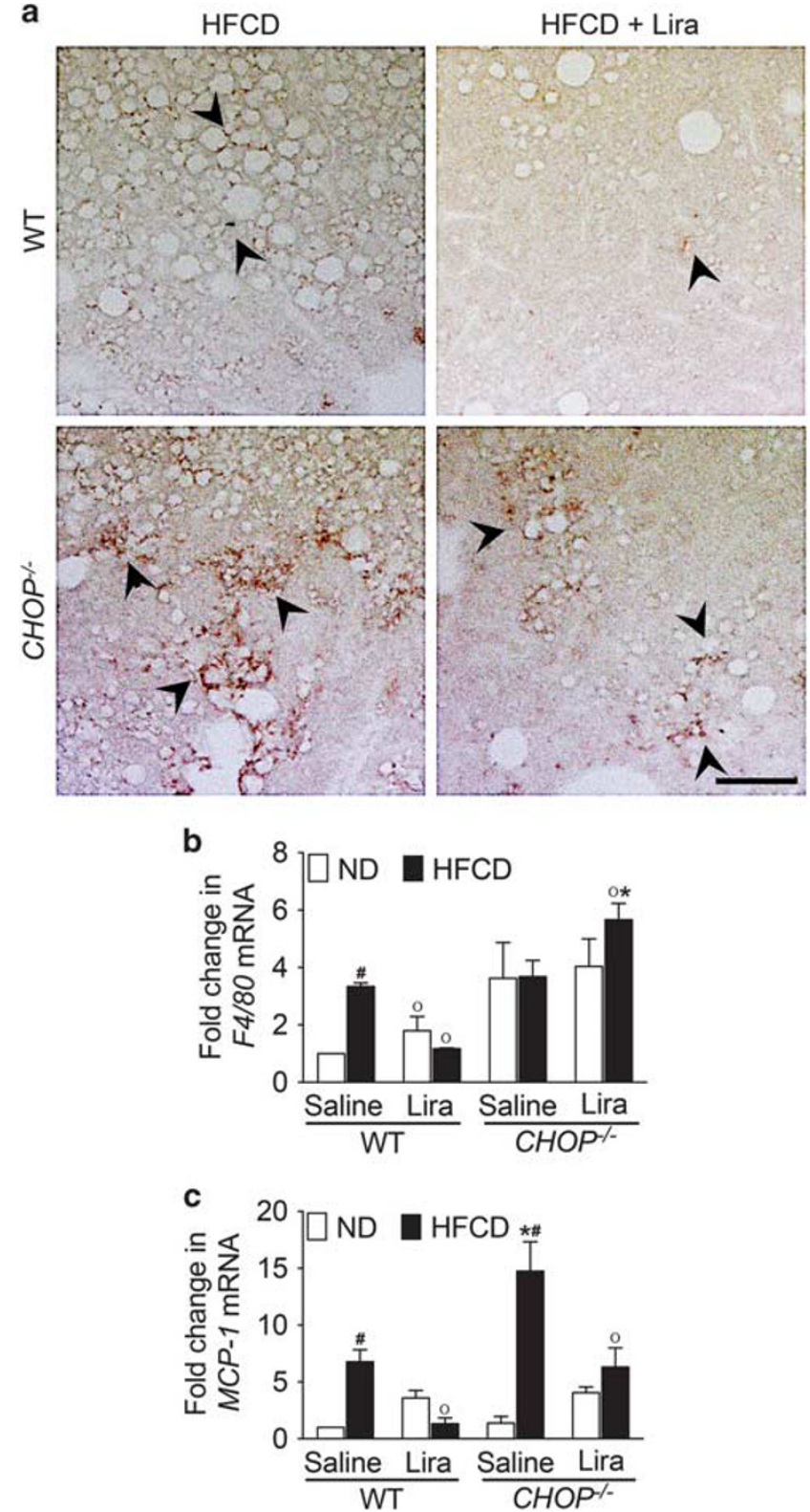

Figure 3 Hepatic macrophage infiltration is higher in HFCD-fed $\mathrm{CHOP}^{-/-}$ mice. (a) Immunohistochemical staining of $\mathrm{F} 4 / 80^{+}$macrophages in the liver. Black arrows, $\mathrm{F} 4 / 80^{+}$macrophages. Quantitative RT-PCR analysis of (b) F4/80 and (c) monocyte chemoattractant protein-1 mRNA transcript levels in the liver of WT or $\mathrm{CHOP}^{-/-}$mice fed a ND or HFCD for 16 weeks. After 12 weeks of feeding, mice received daily injections of saline or liraglutide (Lira) for 4 weeks. Data are presented as mean \pm s.e.m.; $n=5-7$ mice per group. Hashtags indicate significant differences $(P<0.05)$ between ND- or HFCD-fed $\mathrm{CHOP}^{-/-}$mice. Stars indicate significant differences $(P<0.05)$ between WT and $\mathrm{CHOP}^{-1-}$ mice. 'o' indicate significant differences $(P<0.05)$ between saline or liraglutide treated $\mathrm{CHOP}^{-/-}$mice. Scale bar, $20 \mu \mathrm{m}$.

attenuated in both WT and $\mathrm{CHOP}^{-/-}$mice fed the HFCD. Although we did not observe any significant differences in JNK activation between ND- and HFCD-fed WT mice, HFCD significantly increased JNK activation in $\mathrm{CHOP}^{-/-}$mice, which also correlated with impaired insulin sensitivity in these mice (Figure $4 \mathrm{e}$ and g). In agreement with our previous in vitro results, ${ }^{34}$ liraglutide treatment significantly activated $\mathrm{PDK}, \mathrm{AKT}$, and PKC $\zeta$ and reduced JNK activation in HFCD-fed WT mice (Figure 4e-j). Interestingly, liraglutide treatment also induced activation of PDK, AKT, and $\mathrm{PKC} \zeta$ in HFCD-fed $\mathrm{CHOP}^{-/-}$mice. However liraglutide administration in $\mathrm{CHOP}^{-/-}$mice failed to suppress JNK activation; and, although insulin signaling elements were activated, glucose handling in the $\mathrm{CHOP}^{-/-}$mice fed the HFCD was not improved.

\section{Liraglutide Treatment Fails to Improve Lipid, Cholesterol and Glucose Metabolism in the Absence of CHOP}

Next, to understand the discrepancy between insulin signaling pathway activation and failure to improve glucose handling following liraglutide administration in $\mathrm{CHOP}^{-/-}$mice, we investigated the effect of liraglutide on key mediators of lipid, cholesterol, and glucose metabolism. As seen in Figure 5a-d, HFCD significantly increased peroxisomal $\beta$-oxidation and uptake of long-chain fatty acids as indicated by increased expression of carnitine palmitoyltransferase (CPT)-1 $\alpha$, AcylCoA oxidase (ACOX)-1 and ACOX-2, key mediators of peroxisomal $\beta$-oxidation, in WT HFCD-fed mice. HFCD also increased CPT- $1 \alpha$ and ACOX-2 expression in $\mathrm{CHOP}^{-/-}$mice, however the expression level was significantly lower than the WT mice (Figure 5a-d). Liraglutide treatment suppressed $\beta$-oxidation in HFCD-fed WT mice as indicated by increased peroxisome proliferator-activated receptor (PPAR)- $\alpha$ expression and decrease in CPT-1 $\alpha$, ACOX-1, and ACOX-2 expression. None of these key transcription factors and enzymes associated with $\beta$-oxidation were suppressed in the liraglutide administered HFCD-fed $\mathrm{CHOP}^{-/-}$mice, nor was PPAR $\alpha$ increased, suggesting that $\mathrm{CHOP}$ is required for liraglutide-mediated suppression of $\beta$-oxidation (Figure $5 a-d$ ).

In contrast to $\beta$-oxidation, expression levels of sterol regulatory element-binding protein (SREBP)-1c, PPAR- $\gamma$, fatty acid synthase (FAS) and carbohydrate-responsive element-binding protein (ChREBP), key mediators of de novo lipogenesis and carbohydrate metabolism, were significantly higher in HFCD-fed $\mathrm{CHOP}^{-/-}$mice suggesting a role of $\mathrm{CHOP}$ in regulating carbohydrate and fat metabolism (Figure 5e-h). Liraglutide treatment suppressed de novo lipogenesis in both WT and $\mathrm{CHOP}^{-/-}$mice fed the HFCD as indicated by reduced expression of PPAR- $\gamma$ and FAS (Figure 5e-h). Interestingly, liraglutide failed to suppress SREBP1c and ChREBP in HFCD-fed $\mathrm{CHOP}^{-/}$mice suggesting that $\mathrm{CHOP}$ may have a role in liraglutide-mediated suppression of glucose metabolism. Taken together, these data suggest that $\mathrm{CHOP}$ is required for liraglutide-mediated improvement in lipid, cholesterol, and glucose metabolism even though key players of the hepatocyte insulin signaling pathway were activated in both WT and the $\mathrm{CHOP}^{--1}$ mice fed the HFCD. 

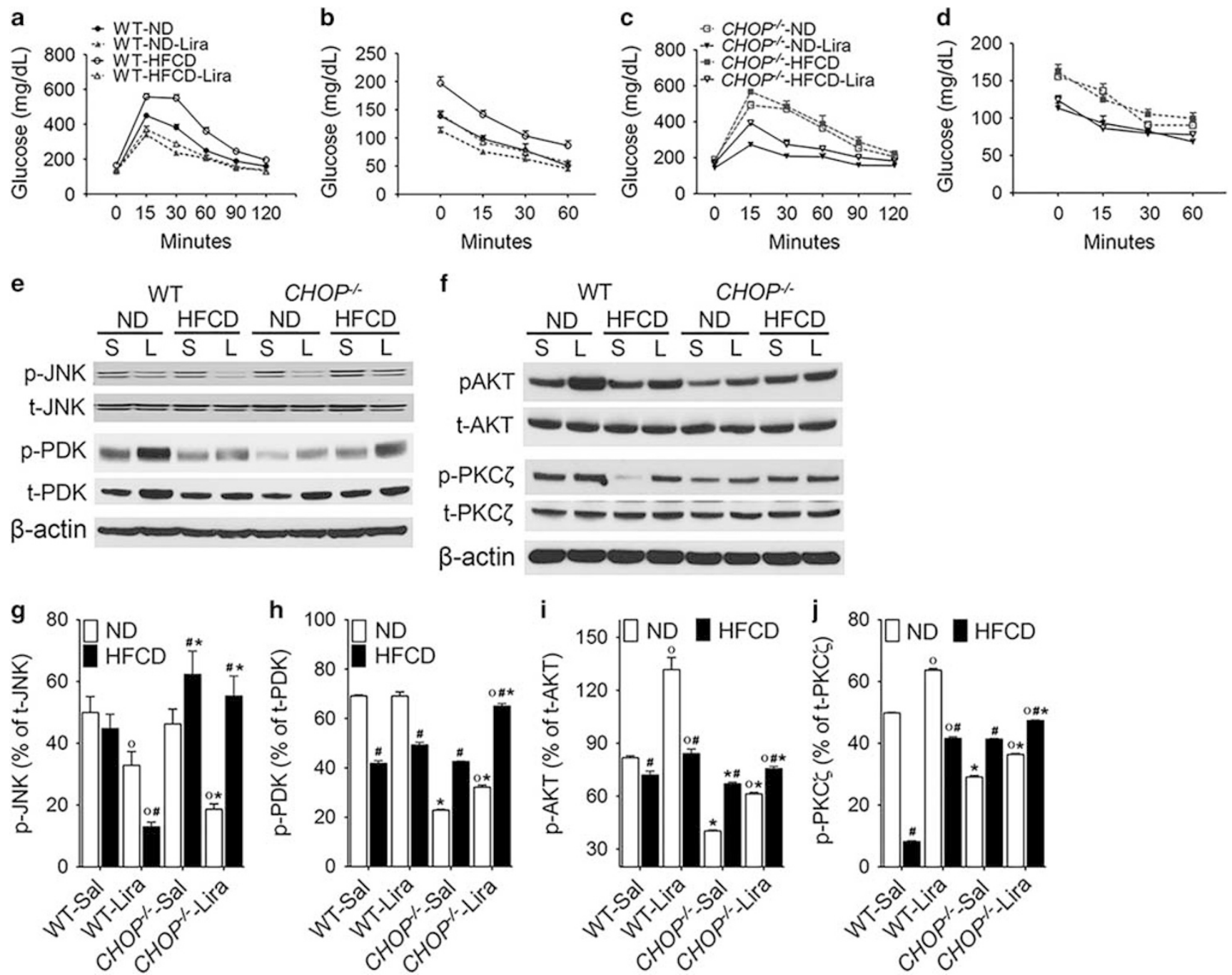

Figure 4 HFCD-fed $\mathrm{CHOP}^{-/-}$mice are resistant to liraglutide-mediated improvement in insulin sensitivity and glucose homeostasis. (a,c) Glucose and (b, d) insulin tolerance in $(\mathbf{a}, \mathbf{b})$ WT and $(\mathbf{c}, \mathbf{d}) \mathrm{CHOP}^{-1-}$ mice fed a ND or HFCD for 16 weeks. After 12 weeks of feeding, mice received daily injections of saline (sal) or liraglutide (Lira) for 4 weeks. (e,f) Representative western blot images and (g-j) densitometry measurements of expression level of various effector molecules of the insulin signaling pathway in the liver. Hashtags indicate significant differences $(P<0.05)$ between ND- or HFCD-fed mice. Stars indicate significant differences $(P<0.05)$ between WT and $C H O P^{-1-}$ mice. 'o' indicate significant differences $(P<0.05)$ between saline- or liraglutidetreated mice. Data are presented as mean \pm s.e.m.; $n=5-7$ mice per group.

\section{Liraglutide Treatment Attenuates ER Stress in HFCD-Fed WT Mice but not in $\mathrm{CHOP}^{-/-}$Mice}

As hepatic ER stress contributes to the development of steatosis, ${ }^{10,11,15,17,45}$ we investigated whether liraglutide treatment attenuates steatohepatitis by suppressing ER stress in the absence of CHOP. As shown in Figure $6 a-h$, HFCD significantly activated PKR-like ER kinase (PERK) and inositol requiring 1 (IRE-1) pathways, as indicated by increased phosphorylation of PERK and its downstream effector E74-like factor 2a (elF2a), and IRE-1 and its downstream effector X-box binding protein (XBP)-1, respectively, in WT mice. HFCD also increased activation of activating transcription factor (ATF)-6 $\alpha$ in WT mice. Similarly all three key UPR pathways were also significantly activated in HFCD-fed $\mathrm{CHOP}^{-/-}$mice (Figure 6a-h).
However, in the absence of CHOP, HFCD induced significantly higher activation of PERK, IRE-1, and ATF- $6 \alpha$ pathways compared with WT control (Figure 6a-h). Liraglutide treatment attenuated HFCD-induced activation of PERK and IRE-1 pathways in WT mice suggesting that liraglutide treatment improved ER homeostasis. However, in HFCD-fed $\mathrm{CHOP}^{-/-}$mice, liraglutide treatment was ineffective in suppressing elF2 and IRE-1 activation, which could account for why liraglutide failed to suppress ER stress in the absence of CHOP. It should be noted that increased activation of IRE-1 in HFCD-fed $\mathrm{CHOP}^{-/-}$mice did not increase XBP-1 activation in these mice. Furthermore, liraglutide treatment significantly increased XBP-1 activation in both WT and $\mathrm{CHOP}^{-/-}$mice fed the HFCD; however, this increase did not correlate with liraglutide-mediated decrease in IRE-1 

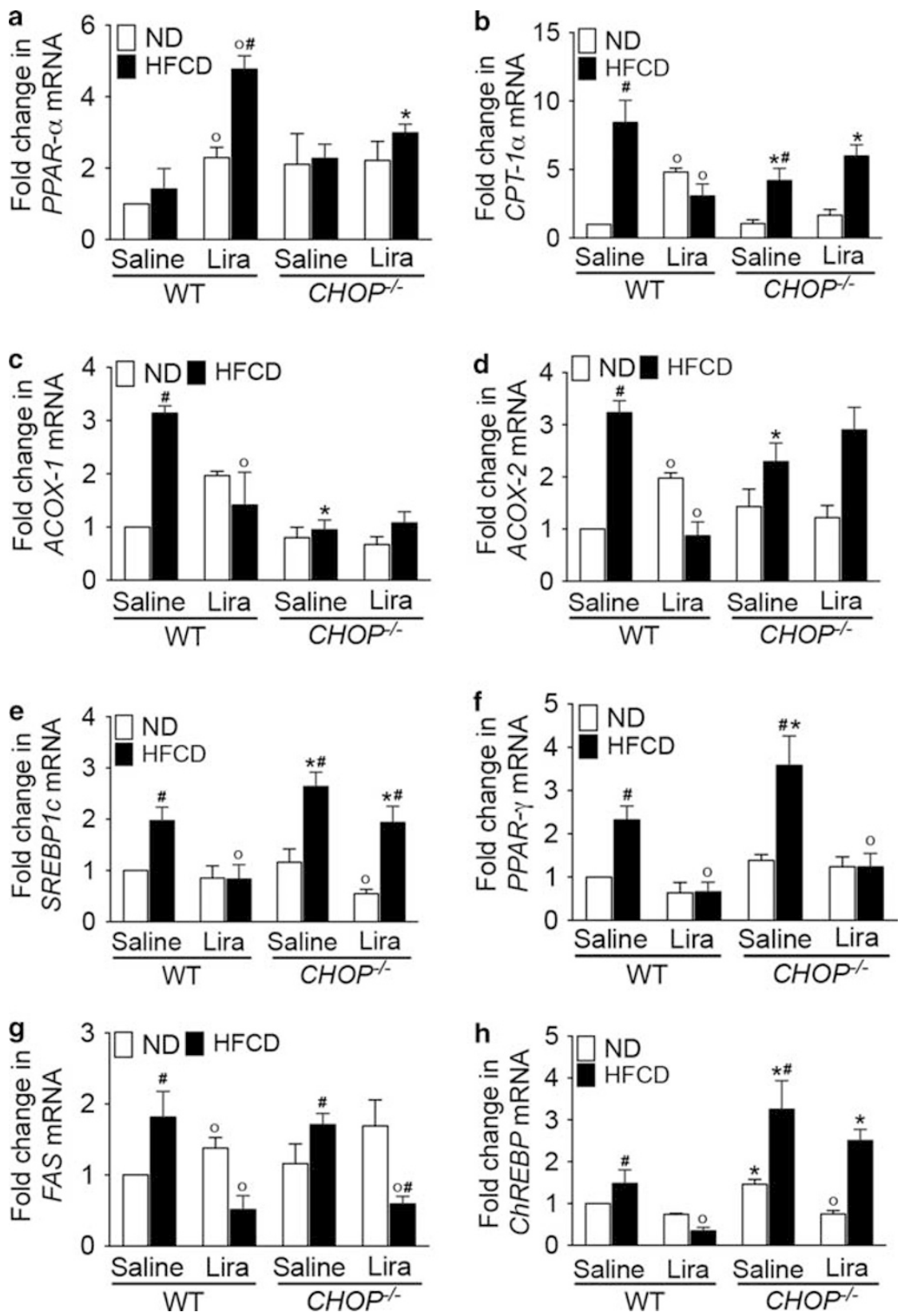

Figure $5 \mathrm{HFCD}$-fed $\mathrm{CHOP}^{-/-}$mice are resistant to liraglutide-mediated improvement in lipid, cholesterol and glucose metabolism. Quantitative RT-PCR analysis of (a) PPAR-a, (b) CPT-1a, (c) ACOX-1, (d) ACOX-2, (e) SREBP1c, (f) PPAR- - , (g) FAS, and (h) ChREBP transcript levels in the liver of WT or CHOP ${ }^{-/-}$ mice fed a ND or HFCD for 16 weeks. After 12 weeks of feeding, mice received daily injections of saline or liraglutide for 4 weeks. Data are presented as mean \pm s.e.m.; $n=5-7$ mice per group. Hashtags indicate significant differences $(P<0.05)$ between ND- or HFCD-fed mice. Stars indicate significant differences $(P<0.05)$ between WT and $\mathrm{CHOP}^{-\prime-}$ mice. 'o' indicate significant differences $(P<0.05)$ between saline- or liraglutide-treated mice.

activation in these mice. We also did not observe any significant differences in GRP78 expression between WT and $\mathrm{CHOP}^{-1-}$ mice fed the HFCD; nor did liraglutide treatment affect GRP78 expression in WT mice fed the HFCD (Figure $6 \mathrm{a}$ and e). However, liraglutide treatment significantly increased GRP78 expression in HFCD-fed $\mathrm{CHOP}^{-/-}$mice. HFCD-induced ER stress in WT mice correlated with increased expression of $\mathrm{CHOP}$, and liraglutide treatment significantly reduced $\mathrm{CHOP}$ expression (Figure 6a and h).
Taken together, these data suggest that liraglutide treatment attenuated HFCD-induced hepatic injury by suppressing ER stress, and implicate a central role for $\mathrm{CHOP}$ in protecting hepatocytes from HFCD-induced ER stress.

\section{Liraglutide Treatment Suppresses Hepatic Apoptosis in HFCD-Fed WT Mice but not in $\mathrm{CHOP}^{-/-}$Mice}

As $\mathrm{CHOP}$ has a role in ER stress-induced apoptosis, and liraglutide treatment has been shown to suppress lipotoxicity- 
a

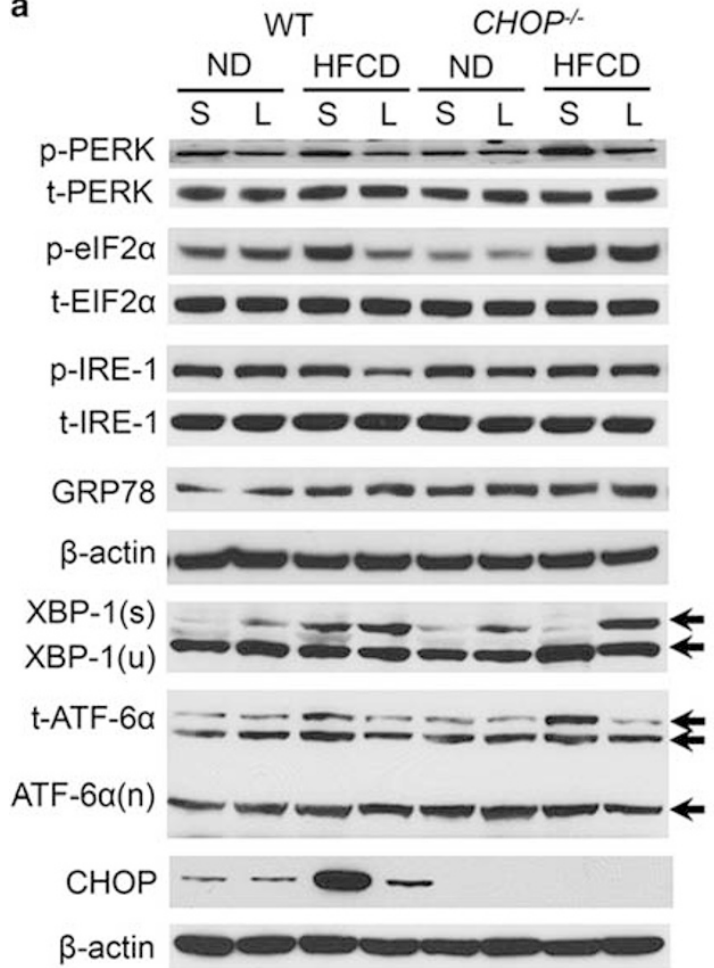

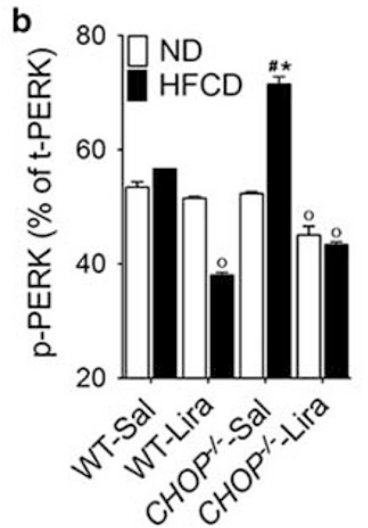

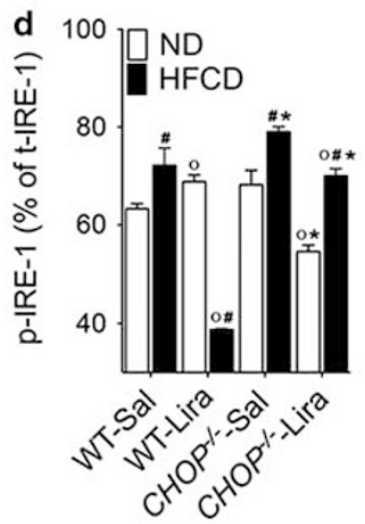

h
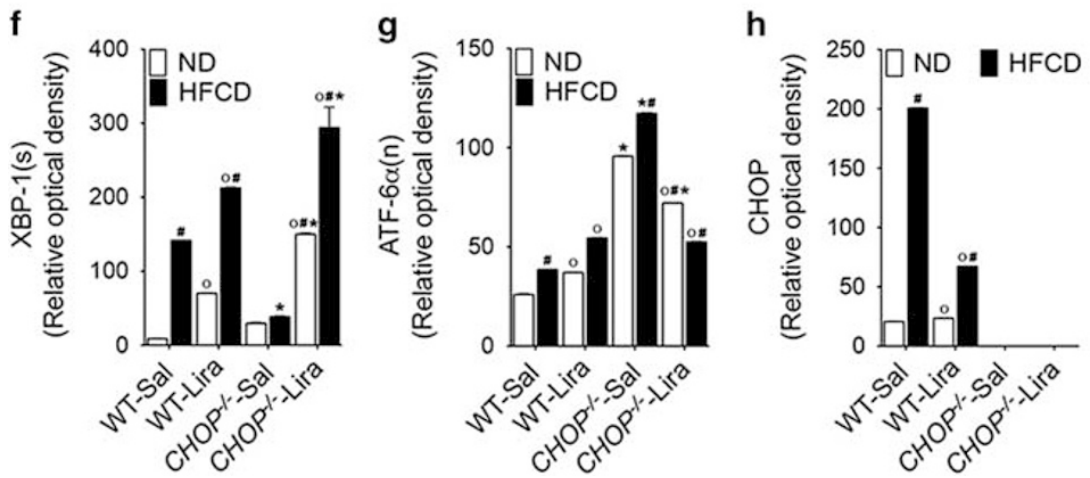
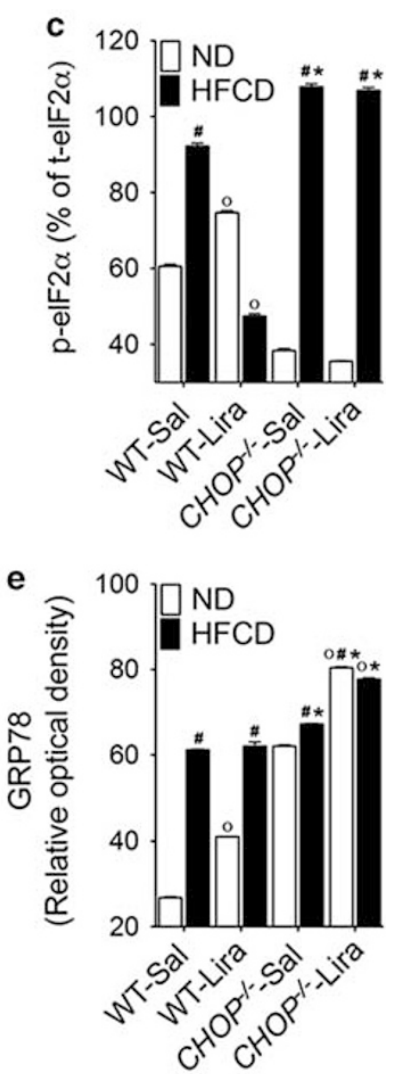

Figure 6 Liraglutide treatment attenuates ER stress in HFCD-fed WT mice but not in $\mathrm{CHOP}^{-/-}$mice. (a) Representative western blot images and (b-h) densitometry measurements of various ER stress parameters in the liver of WT and $\mathrm{CHOP}^{-/}$mice fed a ND or HFCD for 16 weeks. After 12 weeks of feeding, mice received daily injections of saline (sal) or liraglutide (Lira) for 4 weeks. Data are presented as mean \pm s.e.m.; $n=5-7$ mice per group. Hashtags indicate significant differences $(P<0.05)$ between ND- or HFCD-fed mice. Stars indicate significant differences $(P<0.05)$ between WT and $\mathrm{CHOP}^{-/-}$mice. ' $\mathrm{o}$ ' indicate significant differences $(P<0.05)$ between saline- or liraglutide-treated mice.

induced hepatocyte apoptosis, ${ }^{15,17}$ we next investigated whether liraglutide treatment was equally effective in suppressing apoptosis in the absence of CHOP. As seen in Figure 7a-f, HFCD increased hepatocyte apoptosis in both WT and $\mathrm{CHOP}^{-1-}$ mice indicated by increased number of TUNEL-positive cells and caspase-3 activation in the liver. However, absence of CHOP resulted in significantly higher apoptosis in HFCD-fed $\mathrm{CHOP}^{-/-}$mice compared with WT controls (Figure 7a). Higher apoptosis in HFCD-fed $\mathrm{CHOP}^{-/-}$mice correlated with increased $\mathrm{Bax}$ and LC-3B expression, and decreased BCL-2 expression (Figure $7 \mathrm{~b}-\mathrm{f}$ ). Liraglutide treatment reduced apoptosis in both WT and $\mathrm{CHOP}^{-/-}$mice fed the HFCD, however apoptosis remained significantly higher in liraglutide treated HFCD-fed $\mathrm{CHOP}^{-/-}$ mice compared with WT mice fed the HFCD (Figure 7a). While liraglutide treatment significantly lowered caspase-3 activation in $\mathrm{CHOP}^{-/-}$mice, it did not reduce Bax and LC-3B expression in this cohort (Figure $7 \mathrm{~b}-\mathrm{f}$ ). Taken together, these data suggest that $\mathrm{CHOP}$ is critical for liraglutide-mediated suppression of diet-induced hepatocyte death. 

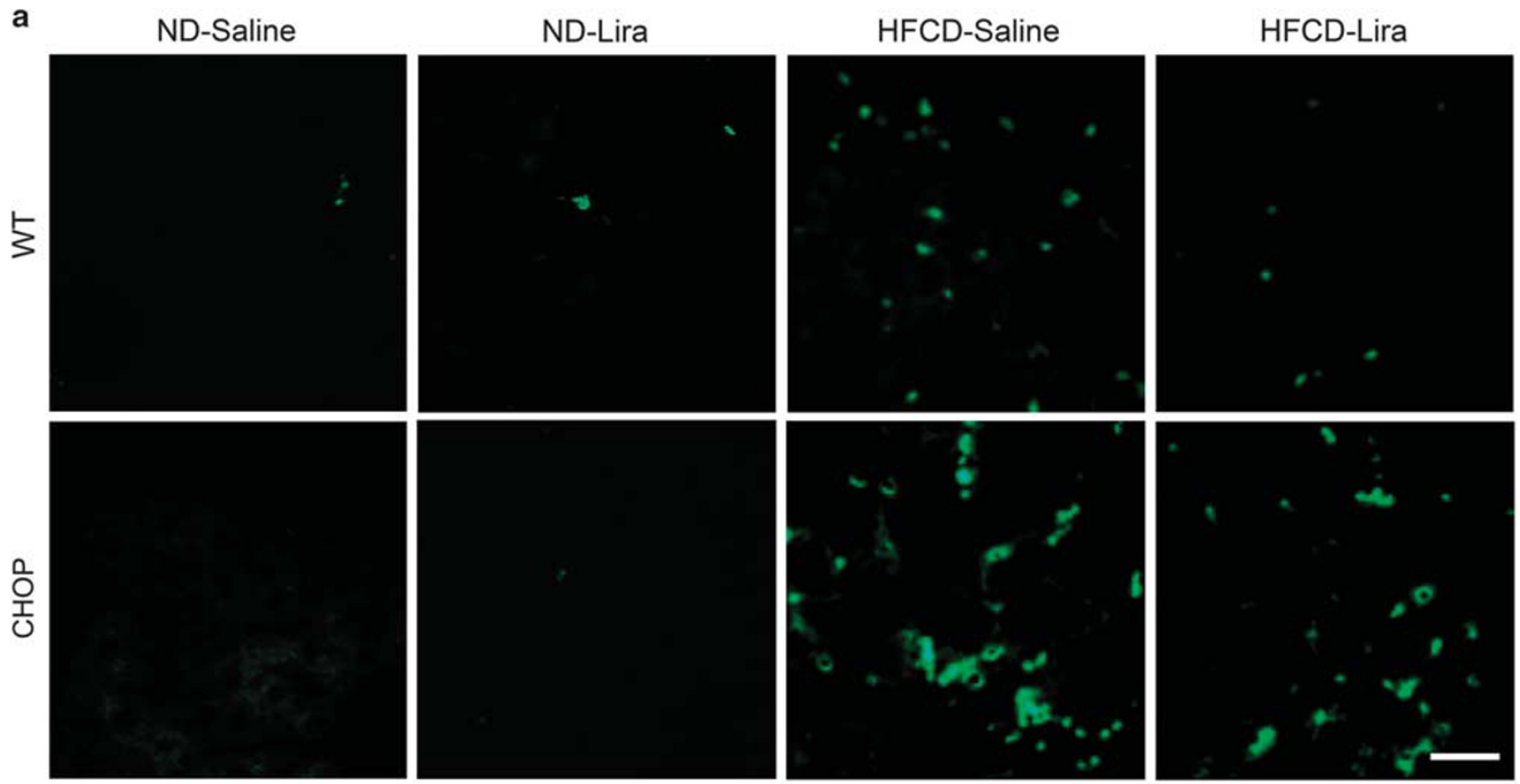

b
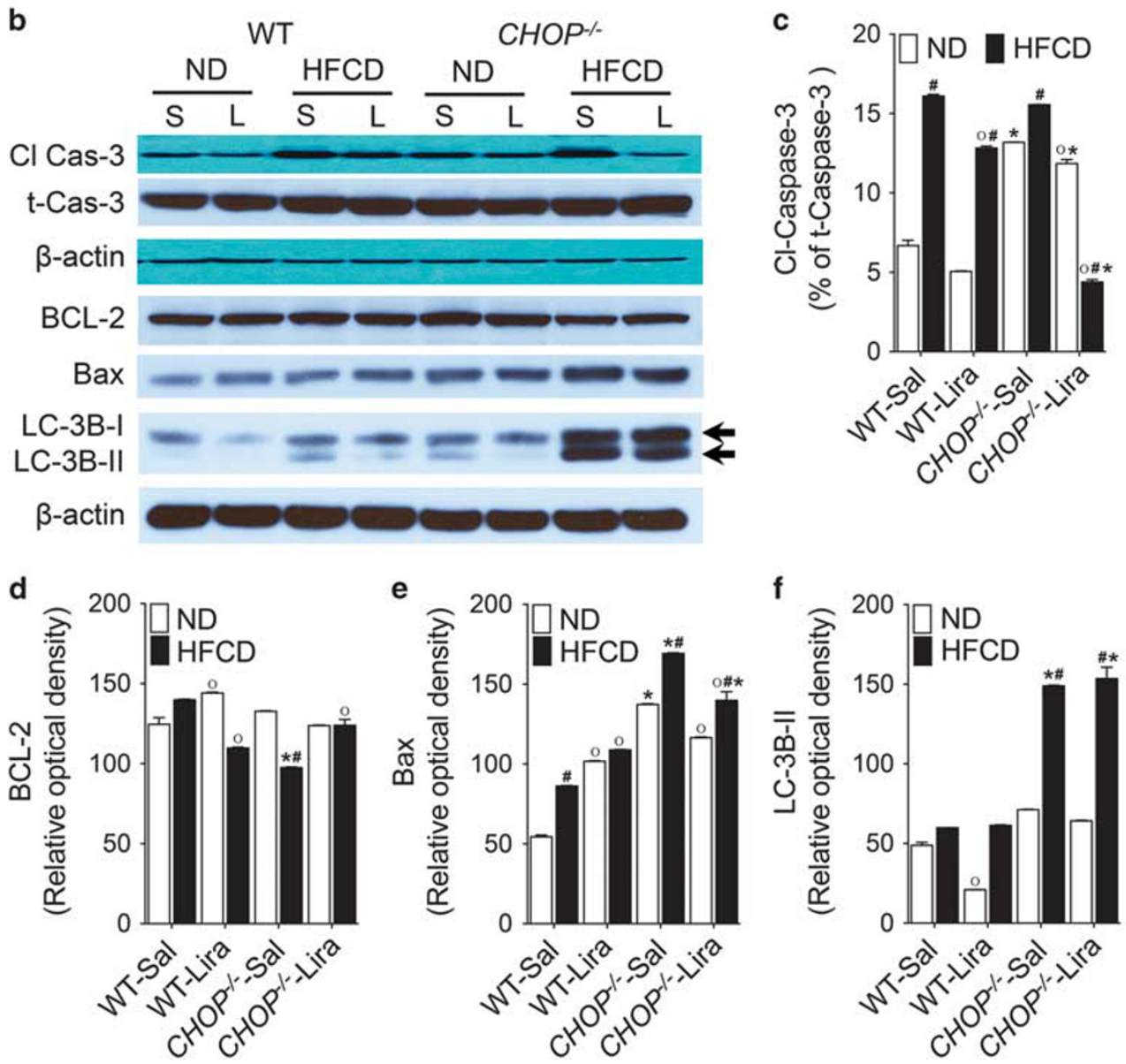
a

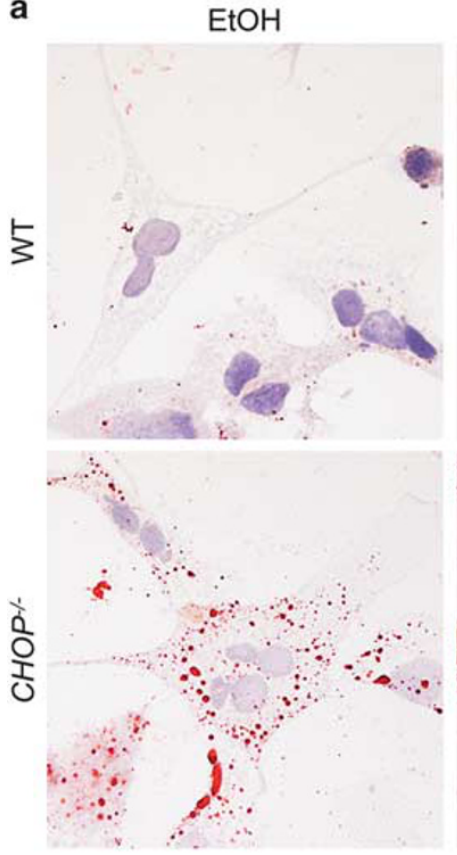

b

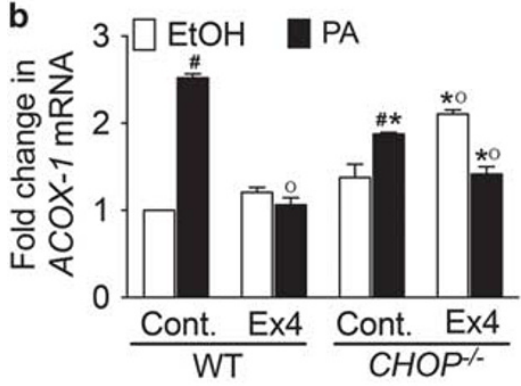

d

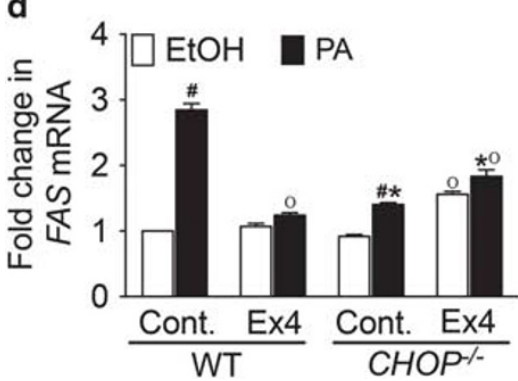

$\mathrm{EtOH}+\mathrm{Ex} 4$

PA

$\mathrm{PA}+\mathrm{Ex} 4$
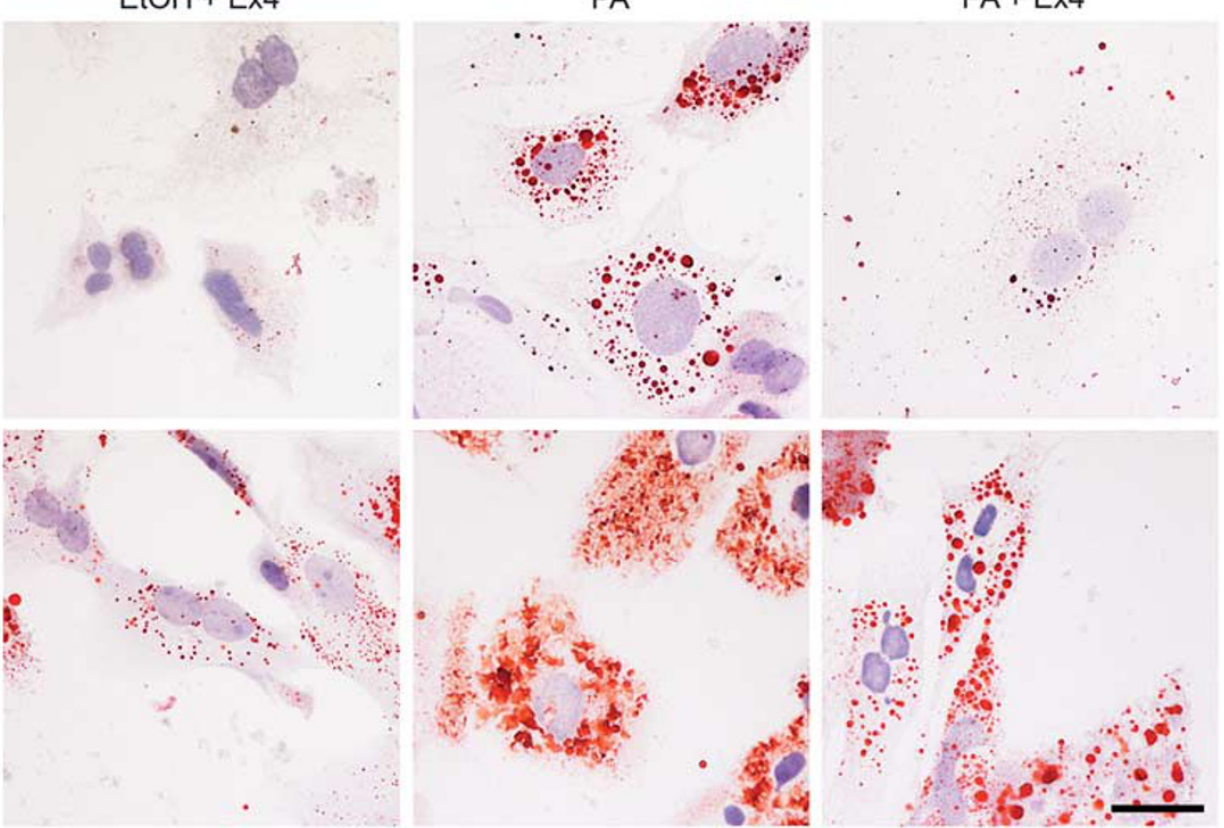

c

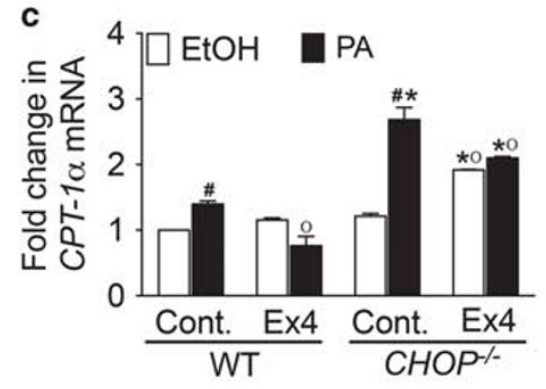

e

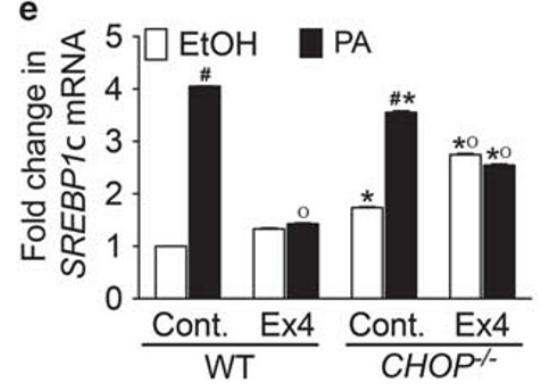

Figure 8 GLP-1 analog exendin-4 attenuates fatty acid deposition in primary hepatocytes from WT mice but not in primary hepatocytes from $\mathrm{CHOP}^{-/-}$mice. (a) Oil Red O-stained micrographs of primary hepatocytes isolated from WT and CHOP ${ }^{-/}$mice. Quantitative RT-PCR analysis of (b) ACOX-1, (c) CPT-1a, (d) FAS, and (e) SREBP1c transcript levels in the primary hepatocytes isolated from WT and $\mathrm{CHOP}^{-/}$mice. Hepatocytes were pretreated with $400 \mu \mathrm{M}$ palmitic acid (PA) in ethanol for $24 \mathrm{~h}$ after which hepatocytes were treated with $10 \mathrm{nM}$ exendin-4 and $400 \mu \mathrm{M}$ PA for an additional $24 \mathrm{~h}$. Hepatocytes treated with ethanol (EtOH), or EtOH plus exendin-4 (Control+Ex4), or PA alone served as controls. Data presented are representative of two independent experiments with four replicates per treatment group. Hashtags indicate significant differences $(P<0.05)$ between EtOH- or PA-treated hepatocytes. Stars indicate significant differences $(P<0.05)$ between WT and $\mathrm{CHOP}^{-1-}$ hepatocytes. 'o' indicate significant differences $(P<0.05)$ between untreated- or liraglutide-treated mice. Scale bar, $10 \mu \mathrm{m}$.

Figure 7 Liraglutide treatment suppresses apoptosis in HFCD-fed WT mice but not in $\mathrm{CHOP}^{-/-}$mice. (a) Representative immunofluorescence images of liver tissue sections stained with terminal deoxynucleotidyl transferase (TUNEL; green) to detect apoptotic cells. (b) Representative western blot images and (c-f) densitometry measurements of hepatic-cleaved caspase-3, BCL-2, Bax and LC-3B expressions in WT and CHOP ${ }^{-/}$mice fed a ND or HFCD for 16 weeks. After 12 weeks of feeding, mice received daily injections of saline (sal) or liraglutide (Lira) for 4 weeks. Data are presented as mean \pm s.e.m.; $n=5-7$ mice per group. Hashtags indicate significant differences $(P<0.05)$ between ND- or HFCD-fed mice. Stars indicate significant differences $(P<0.05)$ between WT and $\mathrm{CHOP}^{-/-}$mice. ' $\mathrm{o}^{\prime}$ indicate significant differences $(P<0.05)$ between saline- or liraglutide-treated mice. Scale bar, $20 \mu \mathrm{m}$. 


\section{GLP-1 Analog Exendin-4 Attenuates Fatty Acid Deposition in Primary Hepatocytes from WT Mice but not in Primary Hepatocytes from $\mathrm{CHOP}^{-/-}$Mice}

To investigate whether the role of $\mathrm{CHOP}$ in modulating liraglutide-mediated protection from NASH in vivo is hepatocyte specific, primary liver hepatocytes isolated from WT and $\mathrm{CHOP}^{-/-}$mice were cultured in $400 \mu \mathrm{M}$ palmitic acid (PA) and treated with $10 \mathrm{nM}$ exendin-4. As seen in Figure 8a, PA treatment significantly increased fat deposition in both WT and $\mathrm{CHOP}^{-/-}$hepatocytes indicated by increased Oil Red O-stained fat droplets in the hepatocytes. It should be noted that fat deposition in the $\mathrm{CHOP}^{-/-}$hepatocytes treated with ethanol alone was higher compared with WT hepatocytes treated with ethanol (Figure 8a). Exendin-4 reduced fat deposition in the PA-treated WT hepatocytes, but failed to reduce fat deposition in PA-treated $\mathrm{CHOP}^{--}$hepatocytes (Figure 8a). Further analysis revealed that PA treatment significantly increased peroxisomal $\beta$-oxidation and uptake of long-chain fatty acids as indicated by increased expression of CPT- $1 \alpha$, and ACOX-1 transcripts in both WT and $\mathrm{CHOP}^{-/-}$ hepatocytes (Figure $8 \mathrm{~b}$ and $\mathrm{c}$ ). Exendin- 4 treatment suppressed $\beta$-oxidation in PA-treated WT hepatocytes, however did not suppress $\beta$-oxidation and fatty acid uptake in PA-treated $\mathrm{CHOP}^{-/-}$hepatocytes (Figure $8 \mathrm{~b}$ and c). Additionally, exendin- 4 also failed to suppress de novo lipogenesis and glucose metabolism in the absence of $\mathrm{CHOP}$ as indicated by significantly higher expression of FAS and SREBP1c transcripts in $\mathrm{PA}+\mathrm{Ex} 4$ treated $\mathrm{CHOP}^{-/-}$hepatocytes (Figure $8 \mathrm{~d}$ and e). Together these data support our in vivo observations and demonstrate that $\mathrm{CHOP}$ has a role in modulating GLP-1 mediated protection from fat deposition in hepatocytes.

\section{DISCUSSION}

In the present study, we demonstrate a critical role for $\mathrm{CHOP}$ in regulating diet-induced ER stress in the setting of administration of the long-acting GLP-1 analog liraglutide in vivo, along with confirmatory in vitro studies in primary hepatocytes derived from $\mathrm{CHOP}^{-/-}$mice. Here, we show that $\mathrm{CHOP}$ has a protective role in reducing ER stress-induced hepatocyte apoptosis, and is essential for the action of liraglutide in mediating these events during nutrient excess. Furthermore, our results demonstrate the contribution of liraglutide mediation of cell carbohydrate and lipid metabolism in preventing disease progression.

$\mathrm{CHOP}$ is a bZIP-containing transcription factor, and is a common point of molecular convergence for all three canonical ER stress transducers, IRE-1, PERK, and ATF-6. ${ }^{46,47}$ CHOP is among the highly inducible genes during ER stress and its expression is primarily regulated at the transcriptional level by various components of the UPR pathway. ${ }^{20,48} \mathrm{CHOP}$ is primarily considered a pro-apoptotic transcription factor that mediates ER stress-induced cell death through the regulation of the Bcl-2 family of proteins. ${ }^{46}$ Consequently, $\mathrm{CHOP}$ deficiency provides partial resistance to
ER stress-mediated apoptosis in in vivo models of chemically induced ER stress. ${ }^{46,47}$ However, paradoxically, in our model of nutrient excess, the results indicate that $\mathrm{CHOP}$ has a protective role, and not a deleterious one, in restoring ER homeostasis.

Despite the ongoing controversy surrounding the expression of GLP-1 receptor in the liver, studies conducted in our laboratory, and others, substantiate a direct role for GLP-1 receptor agonists in suppressing hepatic steatosis in both cell culture and animal models. ${ }^{29-31,34}$ It is now well-established that GLP-1 receptor agonists suppress hepatic accumulation of triglycerides not only by promoting insulin sensitivity in fat-loaded hepatocytes, but also by restoring ER homeostasis and reducing subsequent lipotoxicity-mediated hepatocyte death..$^{2-31,34}$ In agreement with these previous reports, liraglutide administration protected WT mice from HFCD-induced steatohepatitis. However, liraglutide treatment did not have a significant effect on HFCD-induced steatohepatitis in $\mathrm{CHOP}^{-/-}$mice. Liraglutide treatment, in the absence of $\mathrm{CHOP}$ failed to protect the deleterious effects of HFCD-induced liver injury and cell death, strongly suggesting that $\mathrm{CHOP}$ has a direct role in liraglutide-mediated protection from HFCD-induced steatohepatitis. Our results are substantiated by in vitro findings of primary hepatocytes derived from $\mathrm{CHOP}^{-/-}$mice, which demonstrate that GLP-1 analogs must have a role in modulating carbohydrate and lipid metabolism in the hepatocyte.

Apart from modulating ER stress associated apoptosis, $\mathrm{CHOP}$ also regulates carbohydrate and lipid metabolism by modulating transcriptional control of master regulators of glucose metabolism, fatty acid synthesis, and cholesterol metabolism..$^{20,28}$ Our data demonstrate a relationship between liraglutide administration and $\mathrm{CHOP}$ mediated regulation of key genes associated with hepatocyte metabolism in the setting of a western diet (HFCD). In the HFCD-fed $\mathrm{CHOP}^{-/}$ cohort, liraglutide administration failed to repress $\beta$-oxidation, and transcriptional regulators of de novo lipogenesis and cholesterol metabolism suggesting $\mathrm{CHOP}$ functions as a transcriptional repressor of metabolic genes during HFCD-induced ER stress. Taken together, these data suggest that $\mathrm{CHOP}$ has a major role in the liraglutide-mediated transcriptional regulation of hepatic metabolism during nutrient excess. Our findings provide a molecular framework in support of the recently reported small-scale clinical trial, which demonstrated liraglutide reduced hepatic insulin resistance and de novo lipogenesis. ${ }^{16}$

Apart from its insulinotropic effect, GLP-1 analogs also increase insulin sensitivity and glucose uptake by skeletal muscle, hepatocytes, and adipose tissue. ${ }^{30,34,49}$ Our results confirm liraglutide-mediated improvement in insulin sensitivity in HFCD-fed WT mice, but this was not true in the $\mathrm{CHOP}^{-/-}$mice fed the HFCD. These data provide additional evidence for a role of $\mathrm{CHOP}$ in regulating glucose and more importantly fatty acid metabolism. Although the insulin signaling pathway was activated in mice given liraglutide in 
the setting of the Western diet, only WT mice had restored glucose tolerance, not the $\mathrm{CHOP}^{-/-}$mice. As anticipated the WT mice fed the HFCD had a reduction in JNK. That alone could explain why there is less caspase- 3 activation and reduced TUNEL staining. This was not the case for the $\mathrm{CHOP}^{-/-}$mice. In both HFCD-fed $\mathrm{CHOP}^{-/-}$cohorts, JNK activity was unchanged, yet caspase- 3 activity and TUNEL staining were suppressed in the $\mathrm{CHOP}^{-/-}$mice fed the HFCD upon liraglutide administration. This would imply that either CHOP regulates the insulin pathway downstream of PDK-1, $\mathrm{AKT}$ and $\mathrm{PKC}-\zeta$, or that $\mathrm{CHOP}$ functions to suppress de novo lipogenesis. A reduction in de novo lipogenesis would explain reduced lipotoxicity, reduced hepatic inflammation, and ultimately reduced hepatocyte apoptosis.

The implication that CHOP is involved in ER stressinduced lipotoxicity stems from strong correlational data in NAFLD.${ }^{50}$ However, rather than attenuating disease progression, genetic ablation of CHOP accelerates NAFLD progression in both HFD and methionine and choline deficient models of NASH ${ }^{44,51}$ The ability of CHOP to transcriptionally modulate fatty acid metabolism may explain its apparent paradoxical protective role during chronic nutrient excess conditions..$^{20,28}$ This conclusion is consistent with our data showing that liraglutide fails to promote insulin sensitivity, reduce steatosis, restore ER homeostasis, and reduce subsequent lipotoxicity-mediated hepatocyte death in the absence of CHOP. Increased hepatocyte death in HFCD-fed $\mathrm{CHOP}^{-/-}$ mice can be attributed to lower expression of anti-apoptotic protein BCL-2 and higher expression of Bax and LC-3B in these mice. In the $\mathrm{CHOP}^{-/-}$mice, liraglutide administration reduced ER stress-related proteins, but not to the level of the WT mice fed the HFCD. Liraglutide treatment also reduced caspase- 3 activation in HFCD-fed $\mathrm{CHOP}^{-/-}$mice but this did not correlate with a decrease in TUNEL-positive cells suggesting that another pathway in vivo must account for higher hepatocyte apoptosis following liraglutide administration. The increased expression of Bax and LC-3B in HFCDfed $\mathrm{CHOP}^{-/-}$mice and inability of liraglutide to attenuate apoptosis by reducing Bax and LC-3B levels in the absence of caspase- 3 activation implies that both caspase-dependent and -independent cell death pathways are at play in the HFCD-fed $\mathrm{CHOP}^{-/-}$mice. Future studies will be required to clarify the mechanisms of caspase independent cell death pathways.

In summary, our data indicate that in vivo administration of liraglutide may be protective against NAFLD disease progression by initially reducing de novo lipogenesis and thus reducing lipotoxicity. As clinical trials continue, further clarification for the molecular mechanisms accounting for the pleotropic role of long-acting GLP-1 analogs in the liver is warranted.

\section{ACKNOWLEDGMENTS}

This work was supported by NIH grant DK062092, VA grant 101BX001746, and funds from Emory University School of Medicine, to FAA.

\section{DISCLOSURE/CONFLICT OF INTEREST}

The authors declare no conflict of interest.

1. Wree A, Broderick L, Canbay A et al. From NAFLD to NASH to cirrhosisnew insights into disease mechanisms. Nat Rev Gastroenterol Hepatol 2013;10:627-636.

2. Mahady SE, George J. Management of nonalcoholic steatohepatitis: an evidence-based approach. Clin Liv Dis 2012;16:631-645.

3. Adams LA, Lymp JF, St Sauver J et al. The natural history of nonalcoholic fatty liver disease: a population-based cohort study. Gastroenterology 2005;129:113-121.

4. O'Grady MJaC JC. Assessing the Economics of Obesity and Obesity Interventions. Available from http://obesitycampaign.org/documents/Study AssessingtheEconomicsofObesityandObesitylntervention.pdf; last accessed on February 2012.

5. Williams CD, Stengel J, Asike Ml et al. Prevalence of nonalcoholic fatty liver disease and nonalcoholic steatohepatitis among a largely middle-aged population utilizing ultrasound and liver biopsy: a prospective study. Gastroenterology 2011;140:124-131.

6. Tetri LH, Basaranoglu M, Brunt EM et al. Severe NAFLD with hepatic necroinflammatory changes in mice fed trans fats and a high-fructose corn syrup equivalent. Am J Physiol Gastrointest Liver Physiol 2008;295:G987-G995.

7. Neuschwander-Tetri BA. Hepatic lipotoxicity and the pathogenesis of nonalcoholic steatohepatitis: the central role of nontriglyceride fatty acid metabolites. Hepatology 2010;52:774-788.

8. Malhi H, Gores GJ. Molecular mechanisms of lipotoxicity in nonalcoholic fatty liver disease. Semin Liver Dis 2008;28:360-369.

9. Li J, Huang J, Li JS et al. Accumulation of endoplasmic reticulum stress and lipogenesis in the liver through generational effects of high fat diets. J Hepatol 2012;56:900-907.

10. Zhou H, Liu R. ER stress and hepatic lipid metabolism. Front Genet 2014;5:112.

11. Malhi H, Kaufman RJ. Endoplasmic reticulum stress in liver disease. J Hepatol 2011;54:795-809.

12. Xu C, Bailly-Maitre B, Reed JC. Endoplasmic reticulum stress: cell life and death decisions. J Clin Invest 2005;115:2656-2664.

13. Walter $P$, Ron $D$. The unfolded protein response: from stress pathway to homeostatic regulation. Science 2011;334:1081-1086.

14. Li Y, Ge M, Ciani L et al. Enrichment of endoplasmic reticulum with cholesterol inhibits sarcoplasmic-endoplasmic reticulum calcium ATPase- $2 b$ activity in parallel with increased order of membrane lipids: implications for depletion of endoplasmic reticulum calcium stores and apoptosis in cholesterol-loaded macrophages. J Biol Chem 2004;279:37030-37039.

15. Oyadomari S, Harding HP, Zhang $\mathrm{Y}$ et al. Dephosphorylation of translation initiation factor 2alpha enhances glucose tolerance and attenuates hepatosteatosis in mice. Cell Metab 2008;7:520-532.

16. Ozcan U, Cao Q, Yilmaz E et al. Endoplasmic reticulum stress links obesity, insulin action, and type 2 diabetes. Science 2004;306:457-461.

17. Wang $D$, Wei $Y$, Pagliassotti MJ. Saturated fatty acids promote endoplasmic reticulum stress and liver injury in rats with hepatic steatosis. Endocrinology 2006;147:943-951.

18. Cnop M, Foufelle F, Velloso LA. Endoplasmic reticulum stress, obesity and diabetes. Trends Mol Med 2012;18:59-68.

19. Szegezdi E, Logue SE, Gorman AM et al. Mediators of endoplasmic reticulum stress-induced apoptosis. EMBO Rep 2006;7:880-885.

20. Chikka MR, McCabe DD, Tyra HM et al. C/EBP homologous protein (CHOP) contributes to suppression of metabolic genes during endoplasmic reticulum stress in the liver. J Biol Chem 2013;288: 4405-4415.

21. Oyadomari S, Koizumi A, Takeda $\mathrm{K}$ et al. Targeted disruption of the Chop gene delays endoplasmic reticulum stress-mediated diabetes. J Clin Invest 2002;109:525-532.

22. Song B, Scheuner D, Ron D et al. Chop deletion reduces oxidative stress, improves beta cell function, and promotes cell survival in multiple mouse models of diabetes. J Clin Invest 2008;118:3378-3389.

23. Ji C, Mehrian-Shai R, Chan C et al. Role of CHOP in hepatic apoptosis in the murine model of intragastric ethanol feeding. Alcohol Clin Exp Res 2005;29:1496-1503. 
24. Silva RM, Ries V, Oo TF et al. CHOP/GADD153 is a mediator of apoptotic death in substantia nigra dopamine neurons in an in vivo neurotoxin model of parkinsonism. J Neurochem 2005;95:974-986.

25. Namba T, Tanaka $K$, Ito $\mathrm{Y}$ et al. Positive role of CCAAT/enhancerbinding protein homologous protein, a transcription factor involved in the endoplasmic reticulum stress response in the development of colitis. Am J Pathol 2009;174:1786-1798.

26. Thorp E, Li G, Seimon TA et al. Reduced apoptosis and plaque necrosis in advanced atherosclerotic lesions of Apoe - / - and Ldlr - / - mice lacking CHOP. Cell Metab 2009;9:474-481.

27. Tsukano $\mathrm{H}$, Gotoh $\mathrm{T}$, Endo $\mathrm{M}$ et al. The endoplasmic reticulum stress-C/EBP homologous protein pathway-mediated apoptosis in macrophages contributes to the instability of atherosclerotic plaques. Arterioscler Thromb Vasc Biol 2010;30:1925-1932.

28. Rutkowski DT, Wu J, Back SH et al. UPR pathways combine to prevent hepatic steatosis caused by ER stress-mediated suppression of transcriptional master regulators. Dev Cell 2008;15:829-840.

29. Yang J, Ao N, Du J et al. Protective effect of liraglutide against ER stress in the liver of high-fat diet-induced insulin-resistant rats. Endocrine 2015;49:106-118.

30. Mells JE, Fu PP, Sharma S et al. Glp-1 analog, liraglutide, ameliorates hepatic steatosis and cardiac hypertrophy in C57BL/6J mice fed a Western diet. Am J Physiol Gastrointest Liver Physiol 2012;302: G225-G235

31. Sharma S, Mells JE, Fu PP et al. GLP-1 analogs reduce hepatocyte steatosis and improve survival by enhancing the unfolded protein response and promoting macroautophagy. PloS one 2011;6:e25269.

32. Liu Y, Wei R, Hong TP. Potential roles of glucagon-like peptide-1-based therapies in treating non-alcoholic fatty liver disease. World J Gastroenterol 2014;20:9090-9097.

33. Ao N, Yang J, Wang $X$ et al. Glucagon-like peptide-1 preserves nonalcoholic fatty liver disease through inhibition of the endoplasmic reticulum stress-associated pathway. Hepatol Res 2015;46:343-353.

34. Gupta NA, Mells J, Dunham RM et al. Glucagon-like peptide-1 receptor is present on human hepatocytes and has a direct role in decreasing hepatic steatosis in vitro by modulating elements of the insulin signaling pathway. Hepatology 2010;51:1584-1592.

35. Drucker DJ. Minireview: the glucagon-like peptides. Endocrinology 2001;142:521-527.

36. Armstrong MJ, Hull D, Guo K et al. Glucagon-like peptide 1 decreases lipotoxicity in non-alcoholic steatohepatitis. J Hepatol 2016;64: 399-408.

37. Mells JE, Fu PP, Kumar P et al. Saturated fat and cholesterol are critical to inducing murine metabolic syndrome with robust nonalcoholic steatohepatitis. J Nutr Biochem 2014;26:285-292.
38. Charlton M, Krishnan A, Viker K et al. Fast food diet mouse: novel small animal model of NASH with ballooning, progressive fibrosis, and high physiological fidelity to the human condition. Am J Physiol Gastrointest Liver Physiol 2011;301:G825-G834.

39. Schneider CA, Rasband WS, Eliceiri KW. NIH Image to ImageJ: 25 years of image analysis. Nat Methods 2012;9:671-675.

40. Kleiner DE, Brunt EM, Van Natta M et al. Design and validation of a histological scoring system for nonalcoholic fatty liver disease. Hepatology 2005;41:1313-1321.

41. Kumar $P$, Smith $T$, Rahman $K$ et al. Adiponectin agonist ADP355 attenuates CCl4-induced liver fibrosis in mice. PloS One 2014;9: e110405.

42. Severgnini M, Sherman J, Sehgal A et al. A rapid two-step method for isolation of functional primary mouse hepatocytes: cell characterization and asialoglycoprotein receptor based assay development. Cytotechnology 2012;64:187-195.

43. Brunt EM, Kleiner DE, Wilson LA et al. Nonalcoholic fatty liver disease (NAFLD) activity score and the histopathologic diagnosis in NAFLD: distinct clinicopathologic meanings. Hepatology 2011;53: 810-820.

44. Malhi H, Kropp EM, Clavo VF et al. C/EBP homologous protein-induced macrophage apoptosis protects mice from steatohepatitis. J Biol Chem 2013;288:18624-18642

45. Jo $\mathrm{H}$, Choe SS, Shin KC et al. Endoplasmic reticulum stress induces hepatic steatosis via increased expression of the hepatic very lowdensity lipoprotein receptor. Hepatology 2013;57:1366-1377.

46. Li Y, Guo Y, Tang J et al. New insights into the roles of CHOP-induced apoptosis in ER stress. Acta Biochim Biophys Sin (Shanghai) 2014;46: 629-640.

47. Oyadomari S, Mori M. Roles of CHOP/GADD153 in endoplasmic reticulum stress. Cell Death Differ 2004;11:381-389.

48. Okada T, Yoshida H, Akazawa $\mathrm{R}$ et al. Distinct roles of activating transcription factor 6 (ATF6) and double-stranded RNA-activated protein kinase-like endoplasmic reticulum kinase (PERK) in transcription during the mammalian unfolded protein response. Biochem J 2002;366:585-594.

49. Ayala JE, Bracy DP, James FD et al. The glucagon-like peptide-1 receptor regulates endogenous glucose production and muscle glucose uptake independent of its incretin action. Endocrinology 2009;150:1155-1164.

50. Ibrahim SH, Kohli R, Gores GJ. Mechanisms of lipotoxicity in NAFLD and clinical implications. J Pediatr Gastroenterol Nutr 2011;53:131-140.

51. Soon RK Jr, Yan JS, Grenert JP et al. Stress signaling in the methioninecholine-deficient model of murine fatty liver disease. Gastroenterology 2010;139:1730-1739. 